Renato da Costa Teixeira (Orcid: 0000-0002-4073-205X) Carlos Alberto Cavalcante Gomes (Orcid: 0000-0003-2581-9568) ${ }^{2}$ Ellen do Socorro Cruz de Maria (Orcid: 0000-0001-8222-1672) Elissa Raissa Siqueira do Nascimento (Orcid: 0000-0002-7819-981X ) ${ }^{2}$ Joyce Lobato da Costa (Orcid: 0000-0003-3688-0444) ${ }^{2}$

Gerson Lopes de Souza Júnior (Orcid: 0000-0001-7772-3483)

Nadja da Fonseca Veloso (Orcid: 0000-0001-5985-5300) ${ }^{1}$

\section{USO DE MEDIDAS DE BIOSSEGURANÇA POR PROFISSIONAL FISIOTERAPEUTA}

\author{
USE OF BIOSAFETY MEASURES BY A PHYSICAL \\ THERAPIST
}

Autor correspondente

Renato da Costa Teixeira

E-mail: teixeirarenato@globo.com

${ }^{1}$ Programa de Pós-graduação Ensino em Saúde na Amazônia. Universidade do Estado do Pará.

${ }^{2}$ Curso de Fisioterapia. Universidade do Estado do Pará.

Trabalho realizado pelo Grupo de Pesquisa em Saúde Funcional e Qualidade de Vida da Universidade do Estado do Pará

Estudo financiado com recursos da Fundação Amazônia de Amparo a Estudos e Pesquisa - FAPESPA (Edital ${ }^{\circ}$ 019/2018 - UEPA - Programa institucional de bolsas de iniciação científica e de desenvolvimento tecnológico e inovação 2018)

\begin{abstract}
RESUMO
Introdução: a exposição ocupacional e o conceito de biossegurança suscitam reflexões por parte dos profissionais, uma vez que estão mais suscetíveis a contrair doenças advindas de procedimentos que envolvem riscos biológicos, químicos, físicos, ergonômicos e psicossociais. Objetivo: investigar as práticas dos profissionais fisioterapeutas acerca da biossegurança e sua interface com os riscos biológicos e acidentes ocupacionais. Métodos: o presente estudo foi de caráter exploratório, analítico-descritivo, disponibilizado por meio de um questionário on-line do Google Forms com seis questões sobre o perfil do profissional e nove questões acerca da biossegurança. Teve como critérios de inclusão fisioterapeutas de todas as regiões do Brasil que aceitassem participar mediante assinatura do Termo de Consentimento Livre e Esclarecido. Foram excluídos aqueles que não preenchessem mais de $30 \%$ do questionário inviabilizando a análise. Resultados: foram armazenadas 212 respostas, às quais indicam que os participantes que pertencem ao Conselho Regional de Fisioterapia e Terapia Ocupacional da $12^{\text {a }}$ Região $(31,1 \%)$ possuem menos de cinco anos de formado (38,3\%), são especialistas (51,7\%), trabalham no setor privado (43,1\%), em clínicas/ambulatório (46,9\%) nas especialidades de traumato-ortopedia $(28,2 \%)$ ou cardiorrespiratória (27,3\%). Demonstraram conhecer e fazer uso dos equipamentos de biossegurança, no entanto, o uso em algumas áreas ainda é preocupante devido aos índices de não utilização. Conclusão: tornam-se imprescindíveis medidas para que sempre seja abordado esse tema, uma vez que estão relacionados tanto com a segurança do paciente quanto do terapeuta, para assim promover um atendimento mais eficiente e humanizado.
\end{abstract}

Palavras-chave: Fisioterapia; Exposição ocupacional; Medidas de segurança.

\begin{abstract}
Introduction: occupational exposure and the concept of biosafety give rise to reflections on the part of professionals, as they are more susceptible to contracting diseases arising from procedures that involve biological, chemical, physical, ergonomic and psychosocial risks. Objective: to investigate the practices of physical therapists about biosafety and its interface with biological risks and occupational accidents. Methods: the present study was exploratory, analytical-descriptive, made available through an online Google Forms questionnaire with six questions about the professional's profile and nine questions about biosafety. The inclusion criteria were physical therapists from all regions of Brazil who agreed to participate by signing the Free and Informed Consent Form. Those who did not fill more than $30 \%$ of the questionnaire were excluded, making the analysis impossible. Results: 212 responses were stored, which indicate that the participants belonging to the Regional Council of Physiotherapy and Occupational Therapy of the 12th Region (31.1\%) have less than five years of graduation (38.3\%), are specialists (51.7\%), work in the private sector (43.1\%), in clinics / ambulatory $(46.9 \%)$ in the trauma-orthopedics $(28.2 \%)$ or cardiorespiratory $(27.3 \%)$ specialties. They demonstrated to know and make use of the biosafety equipment, however, the use in some areas is still worrying due to the non-use rates. Conclusion: measures are essential to always address this issue, since they are related to both the safety of the patient and the therapist, in order to promote more efficient and humanized care.
\end{abstract}




\section{INTRODUÇÃO}

O cuidado com a exposição ocupacional e o conceito de biossegurança foram introduzidos pela comunidade científica, com foco, inicialmente, nos trabalhadores dos laboratórios de análise de material biológico. Por biossegurança, entende-se o conjunto de ações destinadas a prevenir, controlar, mitigar ou eliminar riscos inerentes às atividades que possam interferir ou comprometer a qualidade de vida, a saúde humana e o meio ambiente ${ }^{1}$.

Atualmente, com a globalização e o intenso e crescente fluxo de pessoas -além das informações, conhecimentos e tecnologias -, agentes químicos, biológicos e patogênicos circulam entre as diversas partes do mundo. Esse fenômeno obriga a comunidade internacional a formar consensos, formular políticas e desenvolver estratégias e instrumentos para lidar com as áreas específicas como a biossegurança, a fim de conhecer e minimizar os impactos à saúde da população mundial ${ }^{2,3}$.

No Brasil, apenas em 1995, com a Lei ${ }^{\circ}$ 8.974 e o Decreto ${ }^{\circ} 1.752$, surgiu a biossegurança, a partir da criação da Comissão Técnica Nacional de Biossegurança (CTNBio) vinculada à Secretaria Executiva do Ministério da Ciência e Tecnologia. Atualmente, é regulamentada pela Lei no 11.105 , de 25 de março de 2005, que dispõe sobre a Política Nacional de Biossegurança ${ }^{4}$.

$\mathrm{Na}$ área da saúde, a biossegurança desencadeia reflexões por parte dos profissionais, especialmente dos que trabalham nas áreas críticas dos hospitais, uma vez que estão mais suscetíveis a adquirir doenças advindas de acidentes de trabalho, principalmente envolvendo agentes biológicos e perfurocortantes ${ }^{5}$.

De acordo com a Resolução RDC n 35, área crítica é
Área na qual existe risco aumentado para desenvolvimento de infecções relacionadas à assistência à saúde, seja pela execução de processos envolvendo artigos críticos ou material biológico, para a realização de procedimentos invasivos ou pela presença de pacientes com susceptibilidade aumentada aos agentes infecciosos ou portadores de microrganismos de importância epidemiológica ${ }^{6}$.

Dentre as ações de biossegurança a fim de evitar ou minimizar esses riscos, pode-se destacar a utilização de equipamentos de segurança, que visam proteger o profissional, seja na exposição ou na manipulação de agentes com possível risco de contaminação. Estes são classificados como equipamentos de proteção individual (EPI) e equipamentos de proteção coletiva (EPC), e são regulamentados pela Portaria $\mathrm{n}^{\circ}$ 485, de 11 de novembro de 2005, do Ministério do Trabalho e Emprego, que aprovou a Norma Regulamentadora - NR - 327.

No contexto da biossegurança, a utilização do EPI é um direito do profissional, e a instituição em que este trabalha é obrigada a fornecê-los. Além disso, a instituição também é obrigada a elaborar e implementar o programa de Prevenção de Riscos Ambientais, com o foco para a prevenção da saúde e a integridade dos trabalhadores. Dessa forma, é de fundamental importância que o profissional da saúde utilize os EPI de forma correta, pois, descartáveis ou não, deverão estar à disposição e em número suficiente nos postos de trabalho, de forma que seja garantido o imediato fornecimento ou reposição ${ }^{8}$.

Pacientes atendidos pelos fisioterapeutas, tanto no meio ambulatorial quanto no hospitalar, variam desde atletas a indivíduos imunodeprimidos; devido a isso, os planos de implantação de normas e procedimentos de controle de infecção devem adequar-se a essa grande varie- 
dade de pacientes. Além disso, enfatiza-se que mesmo fazendo o uso de EPI de boa qualidade, se não forem acompanhados das medidas de precaução-padrão (MPP), não desempenharão sua função de proteção, como prever a biossegurança ${ }^{9}$. Essas medidas incluem a lavagem das mãos, o manuseio apropriado de resíduos dos serviços de saúde e imunização, assim como o uso correto dos EPI e dos EPC.

O fisioterapeuta está diretamente exposto a esses riscos, uma vez que utiliza o seu próprio corpo com instrumento de trabalho, ficando suscetível a risco de acidente com equipamentos, cuidados posturais na realização do trabalho $\mathrm{e}$ até cuidados com radiação não ionizante.

Por isso, foi propósito deste estudo investigar as concepções e práticas dos fisioterapeutas acerca da biossegurança e sua interface com os riscos biológicos e acidentes ocupacionais.

\section{METODOS}

O presente estudo foi de caráter exploratório, analítico-descritivo, a fim de conhecer o uso de medidas de biossegurança pelo fisioterapeuta, sendo realizado de acordo as normas éticas de pesquisa envolvendo seres humanos, iniciado após a aprovação do Comitê de Ética em Pesquisa com Seres Humanos da Universidade do Estado do Pará (parecer 2.429.992) e após aceitação dos profissionais em participarem, por meio do Termo de Consentimento Livre e Esclarecido - TCLE.

A proposta foi realizar um estudo com fisioterapeutas devidamente registrados no Conselho Regional de Fisioterapia e Terapia Ocupacional (Crefito) em todo território nacional.

Como critérios de inclusão, todos os fisioterapeutas que aceitassem a participação mediante assinatura do TCLE. Foram excluídos aqueles que não preenchessem mais de $30 \%$ do ques- tionário inviabilizando a análise.

A coleta de dados foi realizada por meio de um questionário on-line pela plataforma Google Forms. Inicialmente, foi enviada uma solicitação de divulgação para o Conselho Federal de Fisioterapia e Terapia Ocupacional (Coffito), para os Crefito e para a Associação Brasileira de Ensino em Fisioterapia (Abenfisio) solicitando que o formulário fosse enviado aos profissionais. $\mathrm{O}$ Google Forms é um método de coleta de dados que garante o anonimato do respondente. Posteriormente, após uma avaliação que apontava baixa adesão à pesquisa, o formulário foi divulgado por intermédio das redes sociais dos pesquisadores em postagens sobre a biossegurança visando estimular o interesse dos seguidores e a divulgação mediante compartilhamento das postagens. O formulário ficou disponível para respostas de abril de 2018 a novembro de 2018. Com três meses de estudo, foi avaliado que a adesão estava baixa (apenas 75 respostas); dessa forma, deu-se um impulso por meio de postagens em redes sociais dos pesquisadores solicitando que não só respondessem como também compartilhassem a pesquisa, tendo-se alcançado um aumento no número de respostas. Do total de formulários respondidos, foram excluídos três por terem deixado de responder a mais de $30 \%$ das questões.

A análise estatística foi feita de maneira descritiva, os dados foram tabelados em uma planilha disponibilizada pelo Google docs. Após isso, foram analisadas as respostas assinaladas em cada pergunta de forma isolada para cada assertiva. 


\section{RESULTADOS E DISCUSSÃO}

Foram armazenadas 212 respostas, em que todos os participantes aceitaram sua adesão na pesquisa e responderam a, no mínimo, $70 \%$ das questões. O perfil dos participantes encontra-se na Tabela 1.

Tabela 1. Perfil dos participantes da pesquisa

\begin{tabular}{|c|c|}
\hline \multicolumn{2}{|c|}{ Crefito em que estão registrados } \\
\hline Crefito 1 & $4,3 \%$ \\
\hline Crefito 2 & $8,1 \%$ \\
\hline Crefito 3 & $24,9 \%$ \\
\hline Crefito 4 & $3,3 \%$ \\
\hline Crefito 5 & $7,2 \%$ \\
\hline Crefito 6 & $1 \%$ \\
\hline Crefito 7 & $2,4 \%$ \\
\hline Crefito 8 & $4,8 \%$ \\
\hline Crefito 9 & $0,5 \%$ \\
\hline Crefito 10 & $3,3 \%$ \\
\hline Crefito 11 & $2,4 \%$ \\
\hline Crefito 12 & $31,1 \%$ \\
\hline Crefito 13 & $0,5 \%$ \\
\hline Crefito 14 & $0 \%$ \\
\hline Crefito 15 & $0,5 \%$ \\
\hline Crefito 16 & $0,5 \%$ \\
\hline Crefito 17 & $0,5 \%$ \\
\hline Sem inscrição no Crefito & $4,8 \%$ \\
\hline \multicolumn{2}{|c|}{ Titulação } \\
\hline Graduado & $24,9 \%$ \\
\hline Especialização / Residência & $51,7 \%$ \\
\hline Mestrado & $19,6 \%$ \\
\hline Doutorado & $3,8 \%$ \\
\hline \multicolumn{2}{|c|}{ Tempo de formado } \\
\hline Até 5 anos & $38,3 \%$ \\
\hline De 6 a 10 anos & $27,3 \%$ \\
\hline De 11 a 15 anos & $16,3 \%$ \\
\hline Acima de 16 anos & $18,2 \%$ \\
\hline \multicolumn{2}{|c|}{ Setor em que trabalha } \\
\hline Público & $34 \%$ \\
\hline Privado & $43,1 \%$ \\
\hline
\end{tabular}




\begin{tabular}{|c|c|c|}
\hline & Autônomo & $30,1 \%$ \\
\hline & Público e privado & $17,2 \%$ \\
\hline & & \\
\hline & Clínica / ambulatório & $46,9 \%$ \\
\hline & Hospital & $36,8 \%$ \\
\hline & Atendimento domiciliar & $33 \%$ \\
\hline 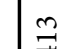 & Docência & $16,7 \%$ \\
\hline & Nasf & $1,9 \%$ \\
\hline & Outro & $11 \%$ \\
\hline$\Xi$ & & \\
\hline$\ddot{\hat{v}}$ & Traumato-ortopedia & $28,2 \%$ \\
\hline กิ & Cardiorrespiratória & $27,3 \%$ \\
\hline 诖 & Neurofuncional & $7,7 \%$ \\
\hline تี & Saúde do idoso & $5,7 \%$ \\
\hline कू & Saúde coletiva & $5,7 \%$ \\
\hline 吾 & Oncologia & $5,3 \%$ \\
\hline ఫृ் & Dermatofuncional & $3,8 \%$ \\
\hline & Saúde da mulher & $2,9 \%$ \\
\hline & Gestão & $1,9 \%$ \\
\hline & Outro & $11,5 \%$ \\
\hline
\end{tabular}

Fonte: elaboração própria.

Com relação às assertivas de biossegurança, $91,9 \%$ afirmaram que estão sujeitos a algum risco biológico em sua atividade profissional, sendo que se destacam como agentes biológicos que podem gerar risco a atividade profissional as NB 2 - Agentes associados a doenças humanas (profilaxia e terapia eficientes) - e as NB 3 Agentes exóticos associados à doenças humanas com potencial para transmissão via aerossol. O Gráfico 1 mostra o percentual de respostas relativas aos agentes biológicos que podem gerar risco, segundo os participantes da pesquisa.

Gráfico 1. Percentual de respostas sobre os agentes biológicos que podem gerar risco para a atividade profissional segundo os participantes da pesquisa.
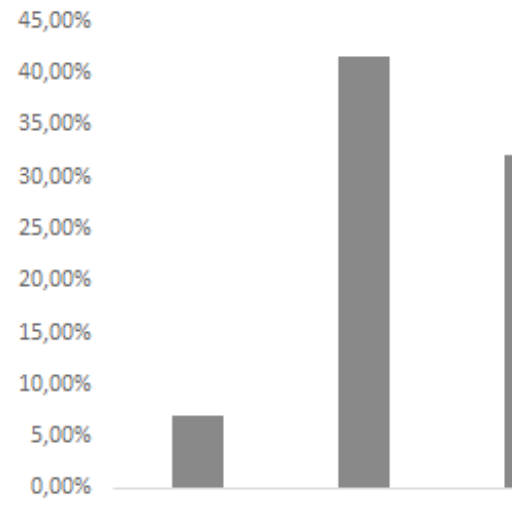

NB1

NB2
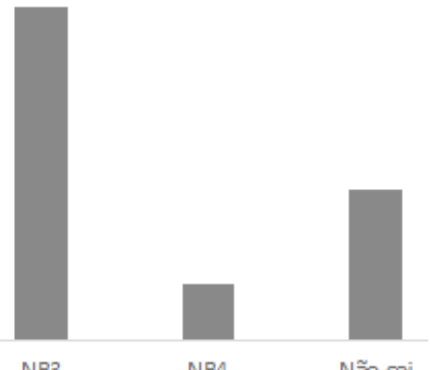

NB1: Agentes que não causam doença; NB2: Agentes associados a doenças humanas (profilaxia e terapia eficiente); NB3: Agentes exóticos associados à doenças humanas com potencial para transmissão via aerossol (profilaxia e terapia limitada); NB4: Agentes letais (humano/ambiente)(Sem profilaxia). Fonte: elaboração própria.
De acordo com as respostas sobre o tipo de exposição envolvendo material biológico que estão sujeitos na atividade profissional, $55,5 \%$ dos participantes acreditam que estão expostos a material biológico, por meio das mucosas, ocorrendo respingos na face envolvendo olho, nariz ou boca ou exposição de mucosa genital. Ressalta-se que, em suas atividades profissionais, o fisioterapeuta tem contato com os três tipos de exposições. As respostas a essa questão encontram-se no Gráfico 2.

Gráfico 2. Tipo de exposição envolvendo material biológico que os participantes acreditam estar sujeitos Fonte: elaboração própria.

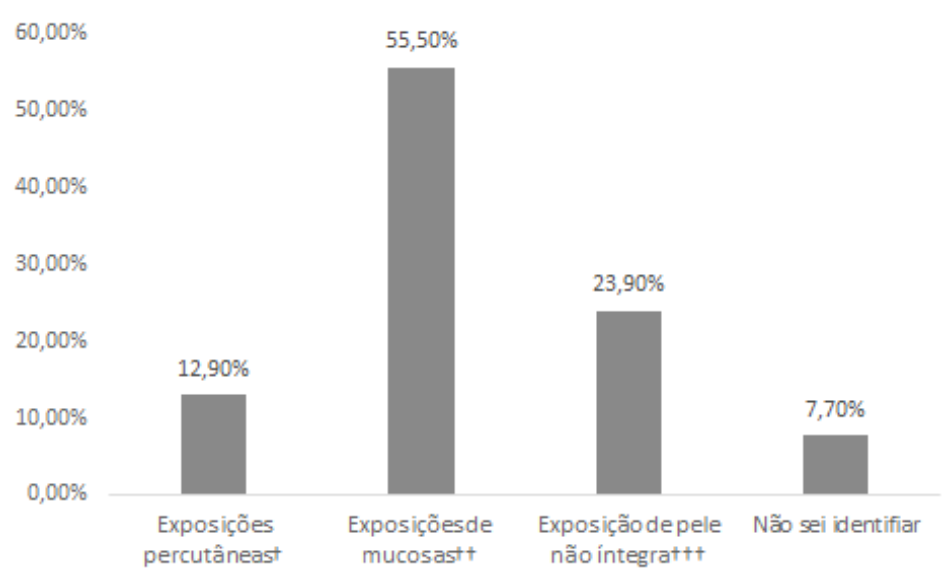

* Exposições percutâneas: lesões provocadas por instrumentos perfurantes ou cortantes; $\dagger \dagger$ Exposições de mucosas: ocorrência de respingos na face envolvendo olho, nariz, boca ou exposição de mucosa genital; ${ }^{\star \star}$ Exposição de pele não íntegra: contato com locais onde a pele apresenta dermatites ou feridas abertas.

Fonte: elaboração própria. 
Nos estudos de Souza ${ }^{10}$ com 38 profissionais de uma equipe multidisciplinar de saúde em uma unidade ambulatorial, que tratou das percepções acerca dos riscos ocupacionais aos quais estão expostos, observou-se que grande parcela dos sujeitos analisa criticamente o processo de trabalho no qual está inserido, na medida em que reconhece que há elevado risco ocupacional em suas atividades laborais, evidenciando que cada categoria profissional enfatizava certo tipo de risco mais do que outros. Os profissionais, porém, reconhecem que existem, no trabalho desempenhado, riscos de diferentes naturezas.

Apesar de saberem que estão sujeitos a risco, ao serem questionados se costumavam utilizar equipamentos de biossegurança, 81,6\% declararam utilizar equipamentos de biossegurança. Dos que não utilizam, 53,7\% afirmaram que "Não está disponível em meu ambiente de trabalho, porém acho que seria necessário"; 35,18\% declararam que "Está disponível, mas não acho necessário"; e 9,26\% disseram que "Não está disponível e não acho necessário". Entre os que responderam que não acham necessário, 92\% declararam trabalhar em clínicas, ambulatórios, atendimento domiciliar, docência e Núcleo Ampliado de Saúde da Família (Nasf).

Essa mesma frequência de utilização dos equipamentos durante suas atividades também foi percebida nos estudos de Rocha ${ }^{11}$, em que a maior parcela dos participantes $(80 \%)$ relatou que utiliza sempre os equipamentos, indepen- dentemente do diagnóstico do paciente, sendo que o restante não se prende ao diagnóstico e utiliza apenas os EPI disponíveis no momento. Os EPI são dispositivos de uso individual destinados a proteger a integridade física e a saúde do trabalhador, sendo indispensável o uso desses equipamentos, por mais simples que seja o procedimento a ser realizado com o paciente, evitando as contaminações cruzadas e preservando a saúde do próprio trabalhador.

Associada a utilização dos EPI, a higienização das mãos antes e após qualquer procedimento é uma prática que contribui para as medidas de biossegurança. No presente estudo, evidenciou-se que a grande maioria costuma higienizar as mãos antes e após o atendimento de seu cliente (94,3\%). Essa é uma das formas mais acessíveis de prevenção da disseminação de infecções, todavia, muitas vezes, é desprezada por muitos profissionais de saúde, sejam estes de grau educacional de nível técnico ou mesmo de nível superior ${ }^{12}$.

Cerca de 93,3\% dos participantes da pesquisa apresentam em seu ambiente de trabalho uma pia para lavagem das mãos antes e após o atendimento do paciente, e $86,1 \%$ possuem um dispenser com álcool em gel para higienização de mãos após o contato com o paciente.

Com relação aos EPI mais utilizados na prática profissional pelos participantes da pesquisa, destacam-se as luvas de procedimento $(78,5 \%)$; o jaleco de tecido (71,3\%); as máscaras $(55,0 \%)$ e a utilização de lixeiras individualizadas (49\%). 
O uso inadequado ou a falta de utilização de medidas de biossegurança pode demonstrar deficit no treinamento e/ou conhecimento dos profissionais; sendo que os principais equipamentos a serem ofertados devem ser: luvas, óculos de proteção, máscara cirúrgica, gorro, avental ou capote e protetor respiratório.

Em estudo ${ }^{13}$ realizado com 67 profissionais da área hospitalar, $84,5 \%$ desses profissionais afirmaram que conhecem o conceito de biossegurança e dos agentes biológicos em sua área de atuação, $\mathrm{e}$ 95,4\% alegaram entender e utilizar os EPI diariamente. Entre esses, os mais empregados foram: luvas $(32,1 \%)$, jaleco $(29,2 \%)$ e máscara $(28,1 \%)$.

Todavia, no estudo realizado por Silva ${ }^{14}$, apenas $47 \%$ afirmaram conhecer o significado do termo biossegurança; e 60\%, a expressão EPI. Em relação, ao uso desse último, os mais utilizados foram: $80 \%$, luva de procedimentos; $80 \%$, jaleco; $52 \%$, sapato fechado; $35 \%$ alegaram que não realizam cursos de atualização sobre a utilização dos EPI e 50\% declararam que não são disponibilizados todos os EPI necessários para atuação dos profissionais.

Nesse sentido, observa-se que a utilização dos tipos de EPI é variável conforme a área de atuação dos profissionais de saúde, contudo, a instituição deve fornecê-los como descrito na literatura garantindo a segurança dos profissionais, os quais devem utilizar os equipamentos de forma adequada para a proteção, permitindo, assim, prevenção de agravos.
Cabe ressaltar que o presente estudo atingiu um menor tamanho amostral do que se supunha conseguir, uma vez que teria como base abranger fisioterapeutas de diferentes regiões do País, o que pode ter influenciado nos seus achados.

Apesar das limitações, observou-se que a uma grande parcela dos fisioterapeutas reconhece a importância das concepções e práticas acerca da biossegurança e sua interface com os riscos biológicos e acidentes ocupacionais.

O fisioterapeuta tem como principal objeto de seu trabalho as suas mãos. $\mathrm{O}$ contato das mãos do profissional fisioterapeuta com o paciente é obrigatório, quer seja por meio de manobras manuais para aliviar a dor, manobras para ajudar no movimento do paciente, manobras para melhorar a circulação periférica ou a elasticidade da pele.

Ao fazermos um cruzamento entre a área de atuação e o uso de equipamentos de biossegurança, percebemos que profissionais que declararam trabalhar em áreas da saúde do idoso, saúde coletiva, traumato-ortopedia têm a maior frequência de não utilização de medidas de biossegurança. Quando comparados entre os profissionais da mesma área, o percentual aumenta nas áreas de saúde do idoso, saúde pública e dermatofuncional. A não utilização dessas medidas coloca em risco a saúde profissional e a do paciente.

Gráfico 4. Frequência de não utilização de equipamento de biossegurança por área de atuação Fonte: elaboração própria.

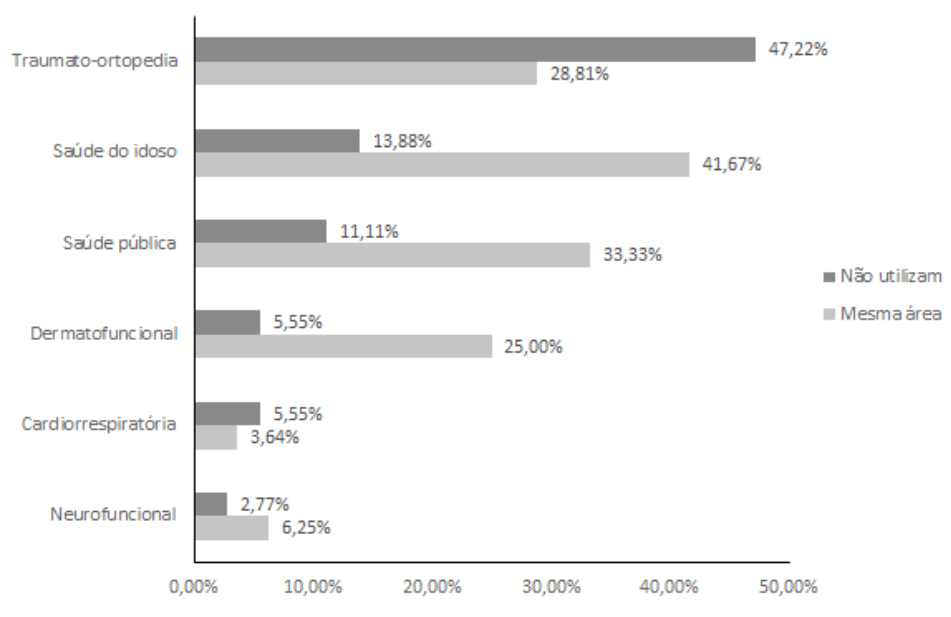


É importante ressaltar que os profissionais de saúde que trabalham em áreas de operação/prestação de cuidados, de emergência, salas cirúrgicas e laboratórios têm um risco maior de exposição, bem como os profissionais de limpeza, de resíduos ou coletores, cujas funções envolvem a manipulação de utensílios contendo material contaminado. A maioria das exposições ocorre nos quartos dos pacientes, além de incidentes em salas de cirurgia, serviços de emergência e unidades de terapia intensiva ${ }^{15}$.

Em um estudo ${ }^{16}$ sobre o conhecimento em Biossegurança por acadêmicos dos cursos de saúde, mostrou que os acadêmicos de Fisioterapia foram os que obtiveram o pior desempenho (39,73\%), mostrando que esses conhecimentos ainda necessitam ser mais trabalhados com os alunos e profissionais dessa área.

O ambiente de atuação do profissional tem sido a principal origem das patologias ocupacionais pela exposição a materiais biológicos aliados à sobrecarga da rotina e às condições de trabalho, sendo fundamental a utilização dos EPI e medidas de proteção para segurança do trabalhador ${ }^{17}$.

É importante ressaltar que há poucos estudos na literatura científica que evidenciam e discorrem sobre o conhecimento de fisioterapeutas sobre a biossegurança, sendo fundamentais, nesse sentido, novas pesquisas que clarifiquem e conscientizem os profissionais para sua melhor atuação.

\section{CONCLUSÃO}

Por meio da análise das respostas acerca da utilização de medidas de biossegurança pelos fisioterapeutas, observa-se que a maior parte dos integrantes da pesquisa conhece e faz uso dessas medidas. Porém, tornam-se fundamentais ações para que sempre seja abordado esse tema, uma vez que estão relacionados tanto com a segurança do paciente quanto do terapeuta, para, assim, promover um atendimento mais eficiente. Sugere-se que novas pesquisas sejam realizadas com maior número de participantes com o objetivo de elaborar medidas preventivas para conscientização sobre a biossegurança, oportunizando promoção da saúde dos profissionais, pacientes e de todos os envolvidos.

\section{REFERÊNCIAS}

1. Brasil. Ministério da Saúde. Biossegurança em saúde: prioridades e estratégias de ação / Ministério da Saúde, Organização Pan-Americana da Saúde. - Brasília: Ministério da Saúde; 2010.

2. Alves LS, Pacheco JS. Biossegurança: Fator determinante nas unidades de atendimento à Saúde. R Flu Exten Univ. 2015;05(1):33-40.

3. Pereira MSC. A biossegurança na prevenção das infecções bacterianas no âmbito hospitalar: revisão de literatura [trabalho de conclusão de curso]. Porto Velho (RO): Centro Universitário São Lucas; 2019.

4. Sousa AFL, Queiroz AAFLN, Oliveira LB, Moura MEB, Batista OMA, Andrade D. Representações sociais da enfermagem sobre biossegurança: saúde ocupacional e o cuidar prevencionista. Rev Bras Enferm. 2016;69(5):864-871.

5. Silva YMP, Araújo VLL, Moura MCL. Saúde do trabalhador em ambiente hospitalar: mapeando riscos e principais medidas de biossegurança. Revista UNINGÁ. 2019;56(2):115-123.

6. Brasil. Ministério da Saúde. Agência Nacional de Vigilância Sanitária. Resolução no 35, de 16 de agosto de 2010. Dispõe sobre o Regulamento Técnico para produtos com ação antimicrobiana utilizados em artigos críticos e semicríticos. Diário Oficial da União. 8 ago 2010. 
7. Brasil. Ministério do Trabalho e Emprego. Portaria 485, de 11 de novembro de 2005. Aprova a Norma Regulamentadora n. 32 (Segurança e Saúde no Trabalho em Estabelecimentos de Saúde). Diário Oficial da União. 16 nov 2005.

8. Macêdo ALGS, Lima LMS, Santos LFO, Galvão B. Influência da biossegurança na assistência à saúde: enfoque na equipe de enfermagem [trabalho de conclusão de curso] [internet]. Recife (PE): Universidade Tiradentes; 2018 [citado 2020 mar 28]. Disponível em: http://openrit.grupotiradentes. com:8080/xmlui/handle/set/2081

9. Sousa FF, Sousa IA, Oliveira LMN. A utilização de equipamentos de proteção individual e coletiva por profissionais de saúde: revisão integrativa. Rev Aten Saúde. 2018;16(58):102-108.

10. Souza NVDO, Pires AS, Gonçalves FGA, Cunha LS, Ribeiro LV, Vieira RS. Riscos ocupacionais e agravos à saúde dos trabalhadores em uma unidade ambulatorial especializada. Rev Min Enferm. 2014;18(4):923-930.

11. Rocha FCS, Meneses R, Cerqueira TCF, Bergamasco MTR, Cacau LAP, Maynard LG, et al. Conhecimento de Biossegurança por Profissionais de Saúde em Unidades Hospitalares. Cadernos de Graduação. 2014;2(1):141-154.

12. Gomes AMP, Martins AR, Carvalho AOC, Pereira MC, Santos PG, Giaretta VMA, Chagas LR. Biossegurança no setor de ortopedia. In: IX Encontro Latino Americano de Iniciação Científica e V Encontro Latino Americanos de Pós Graduação, 2004, São José dos Campos. IX Encontro Latino Americano de Iniciação Científica e V Encontro
Latino Americanos de Pós Graduação, 2004.

13. Lima RJV, Tourinho BCMS, Costa DS, Almeida DMPF, Tapety FI, Landim-Almeida CAP, et al. Agentes biológicos e equipamentos de proteção individual e coletiva: conhecimento e utilização entre profissionais. Rev Pre Infec e Saúde. 2017; 3(1):38-48.

14. Silva JP. Uso dos equipamentos de proteção individual e biossegurança: conhecimento dos auxiliares e técnicos de enfermagem [trabalho de conclusão de curso]. São Luiz (MA): Universidade Federal do Maranhão; 2018.

15. Machado JMH, Assunção AA. Panorama da saúde dos trabalhadores da saúde. Belo Horizonte: UFMG/Faculdade de Medicina; 2012. 164p.

16. Santos PB, Hermes DM, Susin L, Moreira TR. Análise do conhecimento em biossegurança de acadêmicos formandos na área de saúde. Revista UNINGÁ. 2017;53(1):45-50.

17. Santos R, Silva S, Lopes G. Biossegurança em saúde: risco biológico que a equipe de enfermagem está exposta durante sua rotina de trabalho. Anais Concifa; 2018;1(1).
Recebido: 26/08/2019 Aprovado: 11/08/2020 